\title{
Quantifying the Acute Changes in Glucose with Exercise in Type 1 Diabetes: A Systematic Review and Meta-Analysis
}

\author{
Fernando García-García - Kavita Kumareswaran • \\ Roman Hovorka • M. Elena Hernando
}

\begin{abstract}
Background The acute impact of different types of physical activity on glycemic control in type 1 diabetes has not been well quantified.

Objectives Our objective was to estimate the rate of change (RoC) in glucose concentration induced acutely during the performance of structured exercise and at recovery in subjects with type 1 diabetes.

Methods We searched for original articles in the PubMed, MEDLINE, Scopus, and Cochrane databases. Search terms included type 1 diabetes, blood glucose, physical activity, and exercise. Eligible studies (randomized controlled trials and non-randomized experiments) encompassed controlled physical activity sessions (continuous moderate [CONT], intermittent high intensity [IHE], resistance [RESIST],
\end{abstract}

and/or a resting reference [REST]) and reported excursions in glucose concentration during exercise and after its cessation. Data were extracted by graph digitization to compute two $\mathrm{RoC}$ measures from population profiles: $\mathrm{RoC}_{\mathrm{E}}$ during exercise and $\mathrm{RoC}_{\mathrm{R}}$ in recovery.

Results Ten eligible studies were found from 540 publications. Meta-analyses of exercise modalities versus rest yielded the following: $\mathrm{RoC}_{\mathrm{E}}-4.43 \mathrm{mmol} / \mathrm{L} \mathrm{h}^{-1}$ $(p<0.00001,95 \%$ confidence interval $[\mathrm{Cl}]-6.06$ to $-2.79)$ and $\mathrm{RoC}_{\mathrm{R}}+0.70 \mathrm{mmol} / \mathrm{L} \mathrm{h}^{-1}(p=0.46,95 \% \mathrm{CI}$ -1.14 to +2.54 ) for CONT vs. REST; $\mathrm{RoC}_{\mathrm{E}}-5.25 \mathrm{mmol} /$ $\mathrm{L} \cdot \mathrm{h}^{-1}(p<0.00001,95 \% \mathrm{CI}-7.02$ to -3.48$)$ and $\mathrm{RoC}_{\mathrm{R}}$ $+0.72 \mathrm{mmol} / \mathrm{L} \mathrm{h}^{-1}(p=0.71,95 \% \mathrm{CI}-3.10$ to +4.54$)$ for IHE vs. REST; $\operatorname{RoC}_{\mathrm{E}}-2.61 \mathrm{mmol} / \mathrm{L} \mathrm{h}^{-1}(p=0.30$, $95 \% \mathrm{CI}-7.55$ to +2.34$)$ and $\mathrm{RoC}_{\mathrm{R}}-0.02 \mathrm{mmol} / \mathrm{L} \mathrm{h}^{-1}$ $(p=1.00,95 \% \mathrm{CI}-7.58$ to +7.53$)$ for RESIST vs. REST. Conclusions Novel RoC magnitudes $\mathrm{RoC}_{\mathrm{E}}, \mathrm{RoC}_{\mathrm{R}}$ reflected rapid decays of glycemia during CONT exercise and gradual recoveries immediately afterwards. RESIST showed more constrained decays, whereas discrepancies were found for IHE.

\section{Key Points}

Novel glycemia rate-of-change magnitude data expressed in measurable units may provide a means of translating the effects of exercise on glucose dynamics into information that benefits patient self-management.

Rapid decays of glycemia were found during continuous moderate exercise, followed by mild increases immediately afterwards.

Resistance exercise was associated with more constrained decreases, whereas discrepancies were found for intermittent high-intensity exercise. 


\section{Introduction}

Physical activity in type 1 diabetes has complex and dynamic consequences on glucose-insulin regulation. The magnitude of its effect depends on multiple factors, including exercise scheduling, duration and intensity, prior carbohydrate consumption, insulin therapy, pre-exercise glucose levels, and cardiovascular fitness $[1,2]$.

In healthy individuals, exercise stimulates suppression of insulin secretion, resulting in increased hepatic glucose production, lipolysis, and reduced peripheral glucose uptake. In type 1 diabetes, excessive therapeutic insulin levels inhibit hepatic glucose production, which is required to meet the glucose demand by exercising muscles, leading to an increased risk of hypoglycemia [3]. Activation of counter-regulatory hormones, which normally contribute to restoration of glucose levels and triggering of neuroglycopenic symptoms during exercise, is reduced or absent [4]. The behavioral response is subsequently compromised, with resulting failure to recognize symptoms and initiate rescue carbohydrate treatment [5]. The glucose-lowering effects of exercise itself, associated with improved peripheral insulin sensitivity, may persist for several hours and hence contribute to hypoglycemia risk [6].

Continuous exercise of moderate intensity is associated with a greater risk of hypoglycemia in type 1 diabetes [3]. More vigorous activity induces a rise in catecholamines, cortisol, and growth hormone, resulting in hyperglycemia [7]. During recovery in healthy individuals, catecholamines decrease, whereas insulin secretion is increased, resulting in the normalization of glucose levels. In type 1 diabetes, the absence of a rise in endogenous insulin secretion during recovery results in prolonged hyperglycemia [8] and needs specific therapeutic guidelines $[9,10]$. Intermittent highintensity exercise (IHE) may be associated with a lower rate of hypoglycemia than moderate-intensity exercise alone [11]. Resistance exercise (e.g. strength/weight training) has been reported not to alter insulin sensitivity after the performance of exercise [12], which may diminish the occurrence of post-exercise hypoglycemia in type 1 diabetes patients with respect to sustained aerobic activity.

Several studies in literature, for example, Harmer et al. [13], Braken et al. [14], and Kilbride et al. [15], have assessed the impact on glucose levels caused by a range of acute exercise protocols, including different physical activity types. However, there is limited literature comparing these glycemic effects from a quantitative perspective. In this systematic review and meta-analysis, we aim to synthesize quantitatively the acute changes in glucose concentration (and their corresponding rates of variation) induced during exercise sessions and in the subsequent recovery stage, for people with type 1 diabetes.

\section{Methods}

This report adheres to current methodological guidelines on the conduct of systematic reviews for randomized controlled trials (RCT) as in the PRISMA (Preferred Reporting Items for Systematic Reviews and Meta-Analyses) statement [16] and the Cochrane Handbook [17].

\subsection{Eligibility Criteria}

We included studies that enrolled human subjects with type 1 diabetes, regardless of their age or duration of diabetes. Only acute interventions consisting of a standardized exercise protocol with controlled intensity and timing were considered: exercise in free-living conditions and/or prolonged training programs were therefore excluded. As our main outcome of interest was the acute change in glycemic profiles, eligible studies were required to provide measurements reflecting how glucose concentrations evolved over time: from the start of the exercise session until its cessation, and preferably for a period immediately afterwards (early recovery stage). In a first iteration of the review, we restricted our search by study design to incorporate only RCTs. However, given that all of the primarily eligible trials had a crossover design, we decided to also include non-randomized experiments (NREs), i.e. controlled trials where the allocation procedure (order of treatments/interventions) was not random as it is in an RCT. Within-trial comparisons of the main effect of physical activity on glycemia were established either against a control resting period or with respect to profiles from another type of exercise, this depending on the particular design of each study.

\subsection{Study Identification and Selection}

We searched for candidate studies using PubMed, ISI Web of Knowledge's MEDLINE, Scopus, and the Cochrane Library databases. The search was last updated in November 2013. In the first pass, no publication date restriction was set, but due to difficulty in retrieving the full texts of older articles, we decided to limit the range to year 1992 or later. Search terms included type 1 diabetes mellitus, blood glucose, physical activity and exercise as well as their Medical Subject Headings (MeSH) equivalent terms [18], the latter when available in the search engine, i.e. PubMed, MEDLINE, and Cochrane. The full detailed electronic search strategy is shown in the Electronic Supplementary Material (ESM) Appendix S1.

Publications were first screened based on titles and abstracts, and then full contents of candidate papers were examined in depth for a definitive selection. 


\subsection{Data Extraction}

The main features of selected studies were extracted: study design, participant characteristics, full description of the exercise session (type, duration, and intensity), planned food intake, and/or insulin interventions. In order to quantify the degree of variation of glucose concentration over time due to exercise, numeric data about the temporal evolution of glycemia were extracted by digitizing graphs of population mean glucose profiles. To enable analyses independent of the particular physical activity protocol, we defined a magnitude $\mathrm{RoC}_{\mathrm{E}}$ to characterize the mean trend of glucose rate of change (RoC) during the performance of exercise. Magnitude $\mathrm{RoC}_{\mathrm{E}}$ includes (1) the average glucose excursion between the start and the end of the exercise session; and (2) its duration $t_{\mathrm{E}}$. Additionally, in order to assess the mean RoC of glycemia in the early recovery phase (i.e. immediately after exercise termination), we calculated a similar index $\mathrm{RoC}_{R}$ over a recovery interval $t_{\mathrm{R}}$ equal to $30 \mathrm{~min}$ post-exercise. The mathematical definitions of $\mathrm{RoC}_{\mathrm{E}}$ and $\mathrm{RoC}_{\mathrm{R}}$ are as follows:

$$
\begin{aligned}
& \mathrm{RoC}_{\mathrm{E}}=\frac{g_{\mathrm{E}}-g_{\mathrm{O}}}{t_{\mathrm{E}}}=\frac{\Delta g_{\mathrm{OE}}}{t_{\mathrm{E}}} \Rightarrow \\
& \left\{\begin{array}{c}
m\left(\operatorname{RoC}_{\mathrm{E}}\right)=\frac{1}{t_{\mathrm{E}}}\left[m\left(g_{\mathrm{E}}\right)-m\left(g_{\mathrm{O}}\right)\right]=\frac{m\left(\Delta g_{\mathrm{OE}}\right)}{t_{\mathrm{E}}} \\
\mathrm{SD}\left(\operatorname{RoC}_{\mathrm{E}}\right)=\frac{1}{t_{\mathrm{E}}} \sqrt{\mathrm{SD}^{2}\left(g_{\mathrm{E}}\right)+\mathrm{SD}^{2}\left(g_{\mathrm{O}}\right)}
\end{array}\right. \\
& \mathrm{RoC}_{\mathrm{R}}=\frac{g_{\mathrm{R}}-g_{\mathrm{E}}}{t_{\mathrm{R}}}=\frac{\Delta g_{\mathrm{ER}}}{t_{\mathrm{R}}} \Rightarrow \\
& \left\{\begin{array}{c}
m\left(\operatorname{RoC}_{\mathrm{R}}\right)=\frac{1}{t_{\mathrm{R}}}\left[m\left(g_{\mathrm{R}}\right)-m\left(g_{\mathrm{E}}\right)\right]=\frac{m\left(\Delta g_{\mathrm{ER}}\right)}{t_{\mathrm{R}}} \\
\mathrm{SD}\left(\operatorname{RoC}_{\mathrm{R}}\right)=\frac{1}{t_{\mathrm{R}}} \sqrt{\mathrm{SD}^{2}\left(g_{\mathrm{R}}\right)+\mathrm{SD}^{2}\left(g_{\mathrm{E}}\right)}
\end{array}\right.
\end{aligned}
$$

where $g$ represents glycemia measurements: $g_{\mathrm{O}}$ glycemia at the onset of physical activity, $g_{\mathrm{E}}$ glycemia at the end of a given exercise session whose time duration is $t_{\mathrm{E}}$, and $g_{\mathrm{R}}$ is glucose concentration at the end of the recovery period with duration $t_{\mathrm{R}}$ (see Fig. 1 for further details). Similarly, $\Delta g$ denotes total glucose excursions observed between the onset and the end of physical activity $\left(\Delta g_{\mathrm{OE}}\right)$, or between the end of exercise and the termination of recovery phase $\left(\Delta g_{\mathrm{ER}}\right)$. In the formulae, the statistical descriptors sample mean and standard deviation are denoted by $m$ and SD, respectively.

Eligible papers reported mean glucose profiles instead of individualized curves, so no direct information could be obtained regarding the inter-subject statistical variability of the RoCs. To circumvent this issue, we estimated the sample variance for $\mathrm{RoC}_{\mathrm{E}}$ and $\mathrm{RoC}_{\mathrm{R}}$ using the sample variances of glycemia in both extremes of the respective intervals and assuming uncorrelated data (see right-hand side of the equations). Given that this review is the first to

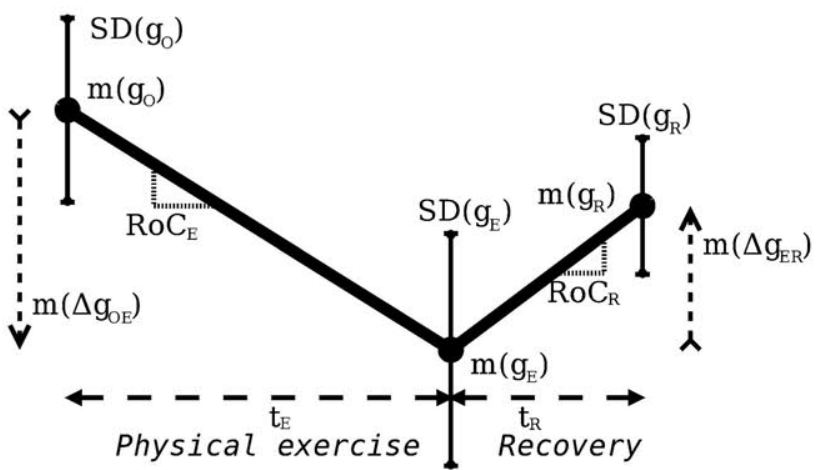

Fig. 1 Schematic depiction of magnitudes employed to describe glycemic profiles and compute rates-of-change $R o C_{E}$ and $R o C_{R}$. Time durations are $t_{\mathrm{E}}$ and $t_{\mathrm{R}}$. Population sample mean and standard deviation values were estimated by graph digitization and entered into the meta-analysis. $g_{O}$ glycemia at the onset of exercise, $g_{E}$ glycemia at the end of exercise, $g_{R}$ glycemia after the recovery period, $m$ mean, $R o C$ rates of change, $S D$ standard deviation, $\Delta g_{O E}$ total glucose excursions during exercise, $\Delta g_{E R}$ total glucose excursions during recovery

estimate RoC values, there is no prior reference in literature about correlations between $g_{\mathrm{O}}, g_{\mathrm{E}}$, and/or $g_{\mathrm{R}}$. Our assumption of uncorrelated measurements may provide a reasonable estimate for the $\mathrm{RoC}$ values to be pooled in the meta-analysis.

\subsection{Statistical Analysis}

Using the DerSimonian and Laird random-effects metaanalysis for difference in means for continuous outcomes, as available in RevMan software [19], we pooled glycemia $R o C_{\mathrm{E}}$ and $\mathrm{RoC}_{\mathrm{R}}$ values across studies and assessed heterogeneity using the $I^{2}$ statistic. We distinguished three types of exercise: continuous physical activity (CONT); IHE, which includes brief bouts of high-intensity sprint-type efforts; and resistance activity (RESIST), e.g. weight lifting.

Comparisons were first established for each exercise modality versus the corresponding resting control periods (REST). Effects of the exercise intervention were computed via within-study differences in means of $\mathrm{RoC}_{\mathrm{E}}$, $\mathrm{RoC}_{R}$ with respect to the glucose RoC at rest. By accounting for the glycemic temporal profile at REST reference and subtracting it out at a study level, i.e. prior to the pooling, we aimed to mitigate background spurious trends attributable to factors other than exercise in the particular protocol of each experiment. Second, comparisons between pairs of exercise types were performed where appropriate studies were available. An exercise modalityCONT in this case-was selected as reference, and withinstudy differences in mean RoCs were calculated with respect to the CONT reference. 


\subsection{Risk of Bias}

To ascertain the validity of candidate publications, we analysed the main indicators for risk of bias in crossover studies [17]: (1) suitability of the crossover design; (2) randomness in the allocation of treatments; (3) presence or absence of carry-over effects; and (4) performing appropriate paired statistical analysis. We assessed publication bias across studies with funnel plots of mean differences to check for possible asymmetries resulting from the nonpublication of trials.

\section{Results}

\subsection{Study Characteristics}

Our electronic search yielded 540 unique references (see Fig. 2), as well as 54 other items discarded due to our publication date criterion (not shown in the figure). Based on a preliminary screening of title and abstract, 148 references were considered potentially relevant. After full text evaluation, we discarded another 131 studies that did not satisfy the pre-specified criteria to be included in our systematic review. The three most frequent reasons for exclusions were (1) observational and other non-RCT/NRE study designs (e.g. case controls, $n=34$ ); (2) studies that comprised a glucose clamp to maintain glycemia artificially stabilized during exercise while measuring other metabolic phenomena (e.g. peripheral insulin sensitivity, $n=24$ ); and (3) studies that investigated the impact of auxiliary interventions apart from exercise itself (e.g. insulin or diet supplement modifications to accommodate exercise, session scheduling, etc., $n=22$ ) in such a way that trial arms focused on the effect of applying or not these side interventions, instead of comparing exercise against either a REST control reference period or versus a different exercise modality.

Of the remaining 17 articles, we decided not to include another eight studies in our meta-analysis due to three reasons that were identified post hoc, namely: (1) in five studies [20-24], patients were supplied with rescue dextrose or carbohydrates, which meant that glucose profiles were artificially altered by these emergency interventions; (2) two studies $[25,26]$ consisted of a single $10 \mathrm{~s}$ sprint at the beginning/end of a session and could not therefore be strictly considered to be either CONT or IHE; and (3) one study [27] did not provide any data about inter-subject variability (mean population profiles only were given). During the process of peer review for this report, journal reviewers identified one extra study that fulfilled our inclusion criteria: Yardley et al. [28]. Table 1 summarizes the main characteristics of the final ten publications [28-37] incorporated in this systematic review and meta-analysis.

Most articles (six of ten) presented glucose data as changes from baseline glycemia, either at the onset of the exercise session [29-31, 33] or at some other reference instant: 90 min before the start of physical activity [35] or $20 \mathrm{~min}$ before the bout [36].

\subsection{Risk of Bias}

Regarding the randomization of treatment allocations, eight of the ten publications had a crossover RCT design where the chronological order of the experimental/control trial arms was set randomly. Yardley et al. [28] did not comment explicitly on randomization in the order of trial arms and, consequently, random order could not be assumed, whereas Yamanouchi et al. [37] employed an NRE design with fixed order of the trial arms, which may have introduced a period effect to some extent.

Table 1 contains a summary of the washout periods in each study protocol, specified by authors in order to avoid (or at least to minimize) the presence of carry-over effects between intervention arms. Most studies required that physical exertions were at least 1 week apart, with some using shorter washouts, although never less than 2 days. Several researchers instructed participants to refrain from any physical activity in the $24-48 \mathrm{~h}$ prior to the test [30, 31,33 ] or to maintain their usual lifestyle [36]. Three publications did not clearly comment on preceding physical activity [28, 29, 35]. In addition, three protocols [30, $32,33]$ checked for the absence of hypoglycemia during the hours or days prior to the exercise sessions and postponed the study in the case of recent hypoglycemic events.

We were unable to compare results from crossover studies against parallel RCTs since none of the latter were found in our literature review. Individually paired statistical analyses were unfeasible here as papers reported only mean glycemic profiles for the study population instead of individual curves for each subject.

The funnel plots (see the ESM, Fig. S1) did not show any evidence of asymmetry that may indicate publication bias. However, the number of studies evaluated was insufficient to allow definitive conclusions to be drawn in this regard.

\subsection{Synthesis of Results and Statistical Analysis}

\subsubsection{Meta-Analysis}

We performed meta-analyses on the within-study differences in $\mathrm{RoC}_{\mathrm{E}}, \mathrm{RoC}_{\mathrm{R}}$ means for each of the three exercise types versus a REST control period, detrending temporal 
Fig. 2 Flow diagram of study selection. $\mathrm{CHO}$ carbohydrates, CONT continuous physical activity, IHE intermittent highintensity exercise, RESIST resistance exercise, REST resting control period

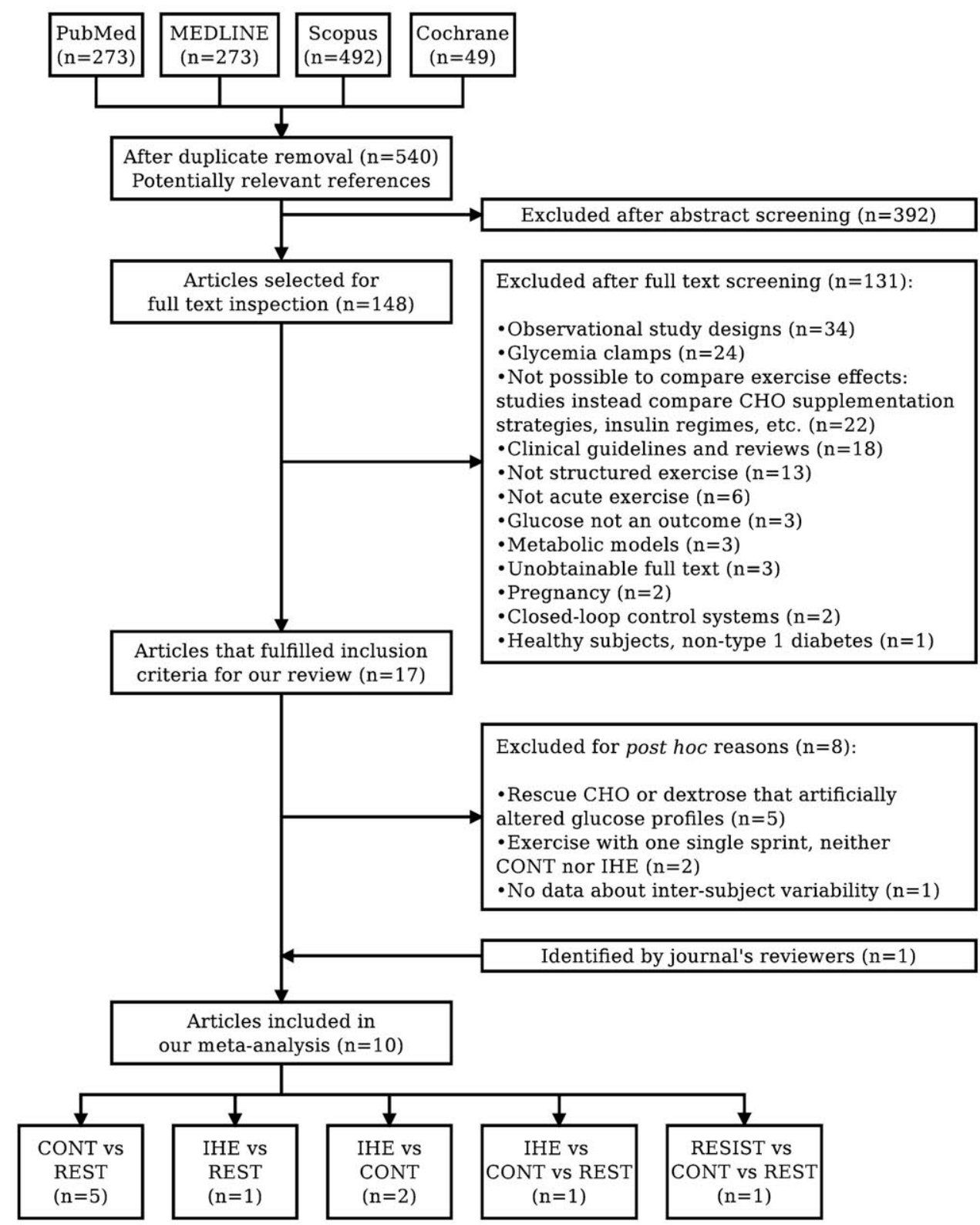

changes with respect to the reference REST glucose profile at a study level prior to the pooling.

Results for CONT versus REST (seven studies and 11 comparisons, see Fig. 3) show that continuous aerobic physical activity at a moderate intensity was associated with significant reductions in glucose concentration during exercise as compared with resting, as well as with a slight rise after exercise cessation that tended to mildly restore glucose levels during recovery. Quantitatively: $\mathrm{RoC}_{\mathrm{E}}$ $\{\mathrm{CONT}$ vs. REST $\}=-4.43 \mathrm{mmol} / \mathrm{L} \mathrm{h}^{-1}(p<0.00001$, $95 \%$ confidence interval (CI) -6.06 to $-2.79 ; I^{2} 41 \%$ ), and $\operatorname{RoC}_{\mathrm{R}} \quad\{\mathrm{CONT}$ vs. REST $\}=+0.70 \mathrm{mmol} / \mathrm{L} \mathrm{h}^{-1}$ $\left(p=0.46,95 \% \mathrm{CI}-1.14\right.$ to $\left.+2.54 ; I^{2} 0 \%\right)$.

Results for IHE versus REST (see Fig. 4) also depicted a pronounced fall in glycemia during physical activity, at a rate of $\operatorname{RoC}_{\mathrm{E}}$ \{IHE vs. REST $\}=-5.25 \mathrm{mmol} / \mathrm{L} \mathrm{h}^{-1}$ $\left(p<0.00001,95 \%\right.$ CI -7.02 to $\left.-3.48 ; I^{2} 0 \%\right)$ when aggregating the two relevant studies. Recovery trends were positive with respect to the resting profiles, although not statistically significant, so: $\operatorname{RoC}_{\mathrm{R}}$ \{IHE vs. REST $\}=$ $+0.72 \mathrm{mmol} / \mathrm{L} \mathrm{h}^{-1}(p=0.71,95 \% \mathrm{CI}-3.10$ to +4.54 ; $\left.I^{2} 0 \%\right)$.

In the case of the RESIST versus REST comparison (see Fig. 5), only one study applied. Outcomes were $\operatorname{RoC}_{\mathrm{E}}$ $\{$ RESIST vs. REST $\}=-2.61 \mathrm{mmol} / \mathrm{L} \mathrm{h}^{-1} \quad(p=0.30$, $95 \% \mathrm{CI}-7.55$ to $+2.34 ; I^{2}$ not applicable); and $\mathrm{RoC}_{\mathrm{R}}$ $\{$ RESIST vs. REST $\}=-0.02 \mathrm{mmol} / \mathrm{L} \mathrm{h}^{-1} \quad(p=1.00$, $95 \% \mathrm{CI}-7.58$ to $+7.53 ; I^{2}$ not applicable).

Additionally, direct comparisons between pairs of exercise modalities were performed when feasible: IHE vs. 
Table 1 Summary of the main characteristics of the ten studies included in our meta-analysis

\begin{tabular}{|c|c|c|c|c|c|c|c|c|c|c|c|c|c|}
\hline \multirow[t]{2}{*}{ Study } & \multicolumn{5}{|l|}{ Population } & \multirow{2}{*}{$\begin{array}{l}\text { Study } \\
\text { design }\end{array}$} & \multirow{2}{*}{$\begin{array}{l}\text { Glucose } \\
\text { samples }\end{array}$} & \multicolumn{3}{|c|}{ Exercise intervention } & \multicolumn{3}{|c|}{ Washout periods } \\
\hline & $\begin{array}{l}\text { Number, sex, } \\
\text { age (years) }\end{array}$ & $\begin{array}{l}\text { Diabetes } \\
\text { duration } \\
\text { (years) }\end{array}$ & $\begin{array}{l}\mathrm{BMI}(\mathrm{kg} / \\
\left.\mathrm{m}^{2}\right)\end{array}$ & $\begin{array}{l}V \mathrm{O}_{2 \max }(\mathrm{ml} / \\
\left.\mathrm{kg} \mathrm{min}^{-1}\right)\end{array}$ & $\begin{array}{l}\mathrm{HbA}_{\mathrm{Ic}} \\
(\%)\end{array}$ & & & $\begin{array}{l}\text { Duration } \\
\text { (min) }\end{array}$ & Description and intensity & $\begin{array}{l}\text { Exercise } \\
\text { types }\end{array}$ & $\begin{array}{l}\text { Between } \\
\text { trial arms }\end{array}$ & $\begin{array}{l}\text { No } \\
\text { exercise } \\
\text { pre-trial }\end{array}$ & $\begin{array}{l}\text { No } \\
\text { hypoglycemia } \\
\text { pre-trial }\end{array}$ \\
\hline $\begin{array}{l}\text { Guelfi et al. } \\
\text { [29] }\end{array}$ & $\begin{array}{l}8, \text { sex NA; } \\
\quad 18.6 \pm 2.1\end{array}$ & $7.0 \pm 4.6$ & $22.1 \pm 1.5$ & $42.4 \pm 7.3$ & $7.0 \pm 0.4$ & RCT & $\begin{array}{l}\text { Capillary } \\
\text { (earlobe) }\end{array}$ & 20 & $\begin{array}{l}\text { Passive recovery with } \\
11 \times 4 \text { s maximal } \\
\text { sprints every } 2 \mathrm{~min}\end{array}$ & $\begin{array}{l}\text { IHE vs. } \\
\text { REST }\end{array}$ & NA & NA & NA \\
\hline $\begin{array}{l}\text { Guelfi et al. } \\
{[30]}\end{array}$ & $\begin{array}{l}4 \mathrm{M}, 3 \mathrm{~F} \\
21.6 \pm 4.0\end{array}$ & $8.6 \pm 5.0$ & $24.7 \pm 3.5$ & $39.3 \pm 7.4$ & $7.4 \pm 1.5$ & $\mathrm{RCT}$ & $\begin{array}{l}\text { Capillary } \\
\text { (earlobe) }\end{array}$ & 30 & $\begin{array}{l}40 \% V_{2 \max } \text { with or } \\
\text { without } 16 \times 4 \mathrm{~s} \\
\text { maximal sprints every } \\
2 \text { min }\end{array}$ & $\begin{array}{l}\text { IHE vs. } \\
\text { CONT }\end{array}$ & 7 days & $24 \mathrm{~h}$ & $48 \mathrm{~h}$ \\
\hline $\begin{array}{l}\text { Iscoe and } \\
\text { Riddell } \\
\text { [31] }\end{array}$ & $\begin{array}{l}5 \mathrm{M}, 6 \mathrm{~F} \\
35.1 \pm 11.6\end{array}$ & $15.6 \pm 18.6$ & NA & $42.4 \pm 5.3$ & $7.8 \pm 1.3$ & RCT & $\begin{array}{l}\text { Interstitial } \\
\text { (CGM) }\end{array}$ & 45 & $\begin{array}{l}55 \% \text { Max load } \\
\left(67.8 \pm 5.0 \% \mathrm{VO}_{2 \max }\right) \\
\text { without or } 50 \% \max \\
\text { load with } 9 \times 15 \mathrm{~s} \\
\text { maximal sprints every } \\
5 \text { min }(68.9 \pm 5.0 \% \\
\left.V \mathrm{O}_{2 \max }\right)\end{array}$ & $\begin{array}{l}\text { IHE vs. } \\
\text { CONT } \\
\text { vs. } \\
\text { REST }\end{array}$ & $\geq 3$ days & $24 \mathrm{~h}$ & NA \\
\hline $\begin{array}{l}\text { Jankovec } \\
\text { et al. [32] }\end{array}$ & $\begin{array}{l}12 \mathrm{M}, 0 \mathrm{~F} \\
33.4 \pm 8.5\end{array}$ & $33.4 \pm 8.5$ & $25.8 \pm 3.7$ & NA & $8.4 \pm 1.0$ & RCT & Blood & $\begin{array}{l}30 \text { (only } \\
1 \text { st } \\
\text { bout) }\end{array}$ & $60 \%$ HR reserve & $\begin{array}{l}\text { CONT } \\
\text { vs. } \\
\text { REST }\end{array}$ & 2 weeks & NA & Previous night \\
\hline $\begin{array}{l}\text { Maran et al. } \\
{[33]}\end{array}$ & $\begin{array}{r}8 \mathrm{M}, 0 \mathrm{~F} \\
34 \pm 7\end{array}$ & $14.3 \pm 8$ & $24 \pm 2.2$ & $33.7 \pm 6.1$ & $7.1 \pm 0.6$ & $\mathrm{RCT}$ & Blood & 30 & $\begin{array}{l}40 \% V \mathrm{O}_{2 \max } \text { with or } \\
\text { without } 15 \times 5 \mathrm{~s} 85 \% \\
V \mathrm{O}_{2 \max } \text { sprints every } \\
2 \mathrm{~min}\end{array}$ & $\begin{array}{l}\text { IHE vs. } \\
\text { CONT }\end{array}$ & $\geq 7$ days & $48 \mathrm{~h}$ & $48 \mathrm{~h}$ \\
\hline $\begin{array}{l}\text { Peter et al. } \\
\text { [34] }\end{array}$ & $\begin{array}{l}12 \mathrm{M}, 1 \mathrm{~F} \\
33.3 \pm 6.5\end{array}$ & $>1$ & $26.8 \pm 3.3$ & NA & $7.6 \pm 1.3$ & $\mathrm{RCT}$ & Blood & 30 & $65.2 \pm 10.1 \% V \mathrm{O}_{2 \max }$ & $\begin{array}{l}\text { CONT } \\
\text { vs. } \\
\text { REST }\end{array}$ & 7 days & $12 \mathrm{~h}$ & NA \\
\hline $\begin{array}{l}\text { Rabasa- } \\
\text { Lhoret } \\
\text { et al. [35] }\end{array}$ & $\begin{array}{l}8 \mathrm{M}, 0 \mathrm{~F} \\
33.0 \pm 8.8\end{array}$ & $12.6 \pm 8.8$ & $23.4 \pm 1.7$ & $37.8 \pm 9.9$ & $6.1 \pm \mathrm{NA}$ & RCT & Blood & 30 or 60 & 25,50, or $75 \% V \mathrm{O}_{2 \max }$ & $\begin{array}{l}\text { CONT } \\
\text { vs. } \\
\text { REST }\end{array}$ & NA & NA & NA \\
\hline $\begin{array}{c}\text { Soo et al. } \\
\text { [36] }\end{array}$ & $\begin{array}{l}8 \mathrm{M}, 1 \mathrm{~F} \\
25.8 \pm 7.4\end{array}$ & $7.3 \pm 6.0$ & NA & NA & NA & $\mathrm{RCT}$ & Blood & 45 & $\begin{array}{l}50 \% \text { HR reserve }(\sim 60 \% \\
\left.V \mathrm{O}_{2 \max }\right)\end{array}$ & $\begin{array}{l}\text { CONT } \\
\text { vs. } \\
\text { REST }\end{array}$ & $\geq 2$ days & $\begin{array}{l}\text { Usual } \\
\text { lifestyle }\end{array}$ & NA \\
\hline $\begin{array}{l}\text { Yamanouchi } \\
\text { et al. [37] }\end{array}$ & $\begin{array}{l}3 \mathrm{M}, 3 \mathrm{~F} \\
\quad 42.7 \pm 13.6\end{array}$ & $5.6 \pm 6.4$ & $20.3 \pm 2.3$ & NA & $7.4 \pm 0.9$ & NRE & Blood & 30 & $\mathrm{HR} \sim 90-110 \mathrm{bpm}$ & $\begin{array}{l}\text { CONT } \\
\text { vs. } \\
\text { REST }\end{array}$ & 2 days & NA & NA \\
\hline $\begin{array}{l}\text { Yardley et al. } \\
{[28]}\end{array}$ & $\begin{array}{l}10 \mathrm{M}, 2 \mathrm{~F} \\
31.8 \pm 15.3\end{array}$ & $12.5 \pm 10$ & NA & $51.2 \pm 10.8$ & $7.1 \pm 1.1$ & NRE & $\begin{array}{l}\text { Blood } \\
\text { (analyzed) } \\
\text { plus } \\
\text { interstitial } \\
\text { (CGM) }\end{array}$ & 45 & $\begin{array}{l}60 \% V_{2 \max } \text { or weight } \\
\text { lifting (intensity not } \\
\text { specified) }\end{array}$ & $\begin{array}{l}\text { RESIST } \\
\text { vs. } \\
\text { CONT } \\
\text { vs. } \\
\text { REST }\end{array}$ & NA & NA & NA \\
\hline
\end{tabular}

Data are expressed as mean \pm standard deviation. Two alternative study designs were encountered, both of them in a crossover design: randomized controlled trials and non-randomized experiments. Three indicators were used to determine washout periods: (1) time elapsed between exercise interventions in the study (i.e. trial arms); (2) whether participants were instructed to refrain from physical activity prior to the experimental session and for how long; and (3) whether researchers checked for the absence of hypoglycemia during the days prior to the exercise sessions

$B M I$ body mass index, $C G M$ continuous glucose monitoring, $C O N T$ continuous physical activity, $F$ female, $H b A_{I c}$ glycated hemoglobin, $H R$ heart rate, $I H E$ intermittent high-intensity exercise, $M$ male, $N A$ not available, NRE non-randomized experiment, $R C T$ randomized controlled trial, RESIST resistance exercise, $R E S T$ resting control period, VO${ }_{2 m a x}$ maximal oxygen uptake 


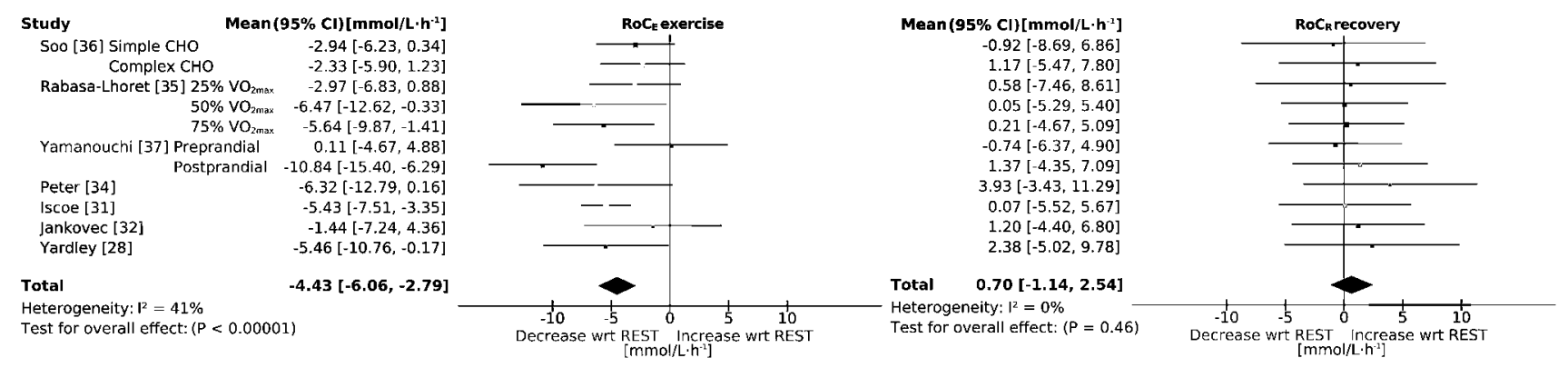

Fig. 3 Overall effect on glycemia profiles of continuous physical activity versus resting control periods. Negative rate-of-change values indicate glucose decaying more rapidly during exercise than in the corresponding resting period; or conversely, increasing more slowly during the recovery stage. $C H O$ carbohydrates, $C I$ confidence interval, CONT continuous physical activity, REST resting control period, $R o C$ rate of change, $R o C_{E}$ glycemia RoC during exercise, $R o C_{R}$ glycemia RoC at recovery, $V O_{2 \max }$ maximal oxygen uptake, wrt with respect to
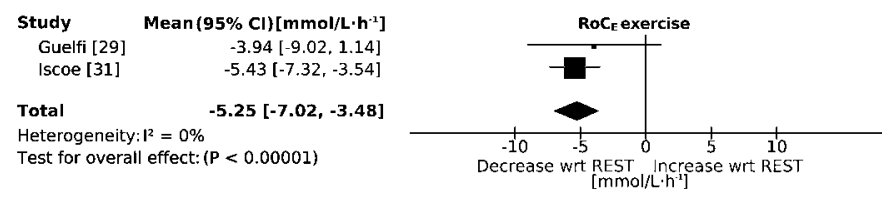

Fig. 4 Overall effect on glycemia profiles of intermittent highintensity exercise versus resting control periods. Negative rates-ofchange values indicate glucose decaying more rapidly during exercise than in the corresponding resting period; or conversely, increasing

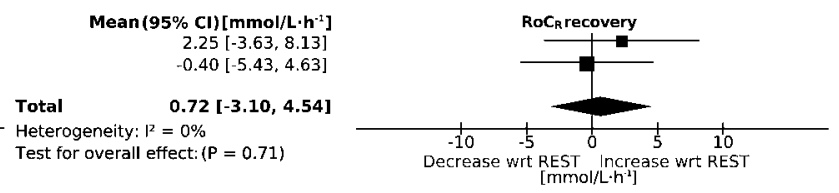

more slowly during the recovery stage. $C I$ confidence interval, $R E S T$ resting control period, $R O C$ rate of change, $R o C_{E}$ glycemia RoC during exercise, $R o C_{R}$ glycemia $\mathrm{RoC}$ at recovery, wrt with respect to
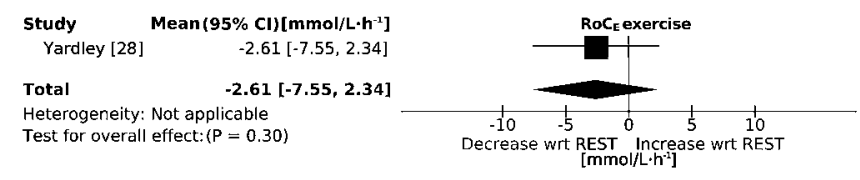

Fig. 5 Overall effect on glycemia profiles of resistance activity versus resting control periods. Negative rate-of-change values indicate glucose decaying more rapidly during exercise than in the corresponding resting period; or conversely, increasing more slowly

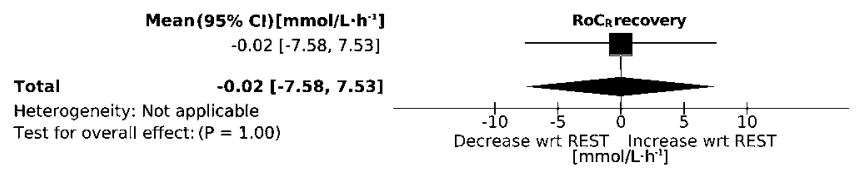

during the recovery stage. $C I$ confidence interval, REST resting control period, $R o C$ rate of change, $R o C_{E}$ glycemia RoC during exercise, $R o C_{R}$ glycemia $\mathrm{RoC}$ at recovery, wrt with respect to
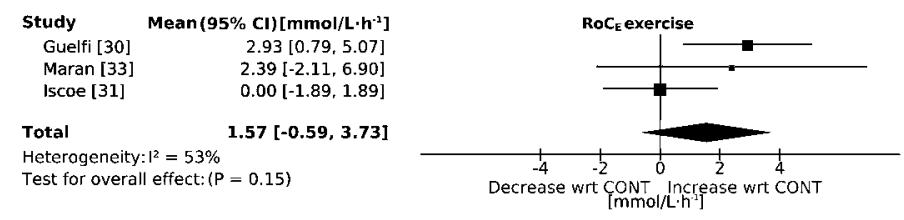

Fig. 6 Difference in the overall effect of intermittent high-intensity exercise versus continuous physical activity. Negative rate-of-change values indicate glycemia decaying more rapidly; or conversely recovering more slowly, in the intermittent high-intensity exercise

CONT based on three studies [30, 31, 33], and RESIST vs. CONT with one study [28]. For the IHE vs. CONT comparison (see Fig. 6), decays in glycemia during exercise were observed to occur less rapidly in the case of IHE, as revealed by a positive $\mathrm{RoC}_{\mathrm{E}}$ value: $\operatorname{RoC}_{\mathrm{E}}\{\mathrm{IHE}$ vs. CONT $\}=$ $+1.57 \mathrm{mmol} / \mathrm{L} \mathrm{h}^{-1}\left(p=0.15,95 \% \mathrm{CI}-0.59\right.$ to $+3.73 ; I^{2}$ $53 \%)$. $\mathrm{RoC}_{\mathrm{R}}$ values were similar: $\mathrm{RoC}_{\mathrm{R}}$ \{IHE vs. CON$\mathrm{T}\}=+0.37 \mathrm{mmol} / \mathrm{L} \mathrm{h}^{-1}(p=0.83,95 \% \mathrm{CI}-2.90$ to $+3.63 ; I^{2} 0 \%$ ). For the RESIST vs. CONT case (see Fig. 7),

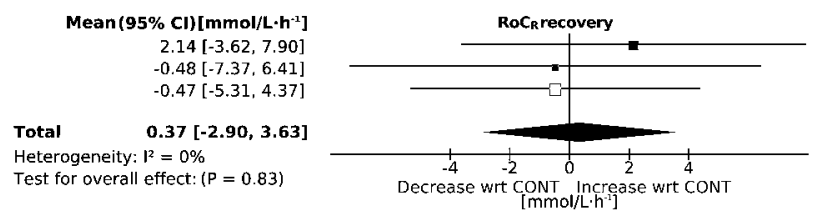

sessions than for the continuous bout. $C I$ confidence interval, CONT continuous physical activity, REST resting control period, $R o C$ rate of change, $R o C_{E}$ glycemia RoC during exercise, $R o C_{R}$ glycemia RoC at recovery, wrt with respect to

Yardley et al. [28] revealed a milder decay of glycemia in RESIST exercise with respect to CONT, as well as slower recovery: $\operatorname{RoC}_{\mathrm{E}}\{$ RESIST vs. CONT $\}=+2.86 \mathrm{mmol} / \mathrm{L} \mathrm{h}{ }^{-1}$ ( $p=0.20,95 \% \mathrm{CI}-1.49$ to $+7.20 ; I^{2}$ not applicable), RoC $_{\mathrm{R}}\{$ RESIST vs. CONT $\}=-2.40 \mathrm{mmol} / \mathrm{L} \mathrm{h}^{-1}(p=$ $0.39,95 \% \mathrm{CI}-7.87$ to $+3.06 ; I^{2}$ not applicable). Of note, Yardley et al. [28] documented negligible fluctuations of glycemia in the RESIST recovery stage in absolute terms, along with positive recoveries in CONT. 

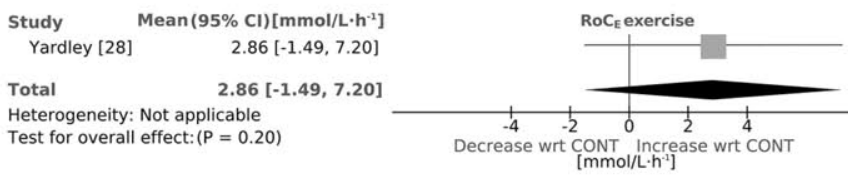

Fig. 7 Difference in the overall effect of resistance activity versus continuous physical activity. Negative rate-of-change values indicate glycemia decaying more rapidly; or conversely recovering more slowly, in the resistance activity sessions than for the continuous

\subsubsection{Meta-Regression}

To ascertain the dose/response influence of varying exercise intensity in terms of RoC values, we carried out a post hoc random-effect meta-regression analysis using metareg package in Stata 13 software (StataCorp LP; College Station, TX, USA). Given the reduced number of studies, this was only feasible for CONT activity. Exercise intensity was measured through $\% V \mathrm{O}_{2 \max }$, i.e. the percentage of a subject's maximal oxygen uptake $\left(V^{2} \mathrm{O}_{2 \mathrm{max}}\right)$. Intensities reported in Jankovec et al. [32] and Soo et al. [36] via heart rate reserve (HRR; 60 and $50 \%$, respectively) were transformed to their equivalent $\% V \mathrm{O}_{2 \max }$ values (55 and $\left.46 \% V_{2 \max }\right)$ based on previous studies [38, 39]. For Yamanouchi et al. [37], we imputed an intensity of $20 \%$ $V \mathrm{O}_{2 \max }$ as corresponding to the range $90-110$ beats per minute [40].

Figure 8a depicts a moderate dependency of $\mathrm{RoC}_{\mathrm{E}}$ with respect to physical activity intensity, with regression slope $-0.0200 \mathrm{mmol} / \mathrm{L} \mathrm{h}^{-1}$ per unit of $\% V \mathrm{O}_{2 \max }$, although not statistically significant $(p=0.69)$. This negative slope manifests more pronounced-i.e. faster-decay rates in glycemia associated with more vigorous CONT exercise in the range of intensities covered by the included studies $\left(20-75 \% \quad V_{2 \max }\right)$; whereas milder exertions produce decays of a lesser absolute magnitude, hence slower glycemic decrements. Conversely, Fig. 8b shows how glycemia tended to recover more rapidly after more vigorous CONT bouts, with a positive regression slope equaling $+0.0117 \mathrm{mmol} / \mathrm{L} \mathrm{h}^{-1}$ per unit of $\% V^{2 \max }(p=0.87$, not significant) in the range of intensities covered by our analysis.

\section{Discussion}

\subsection{Summary of Findings}

This systematic review and meta-analysis aggregated results from ten studies to evaluate the acute impact of various types of structured exercise sessions on the glucoregulatory balance in people with type 1 diabetes. To our knowledge, this is the first published report quantifying the effects on glycemia by means of RoC measures, both

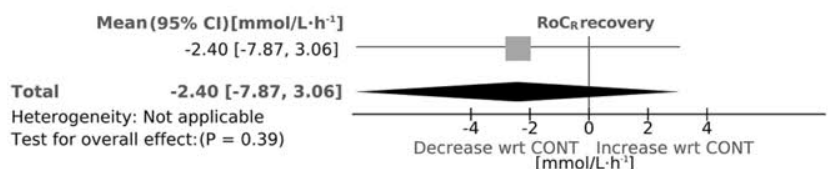

physical activity bout. $C I$ confidence interval, CONT continuous physical activity, $R o C$ rate of change, $R o C_{E}$ glycemia RoC during exercise, $R o C_{R}$ glycemia RoC at recovery, wrt with respect to
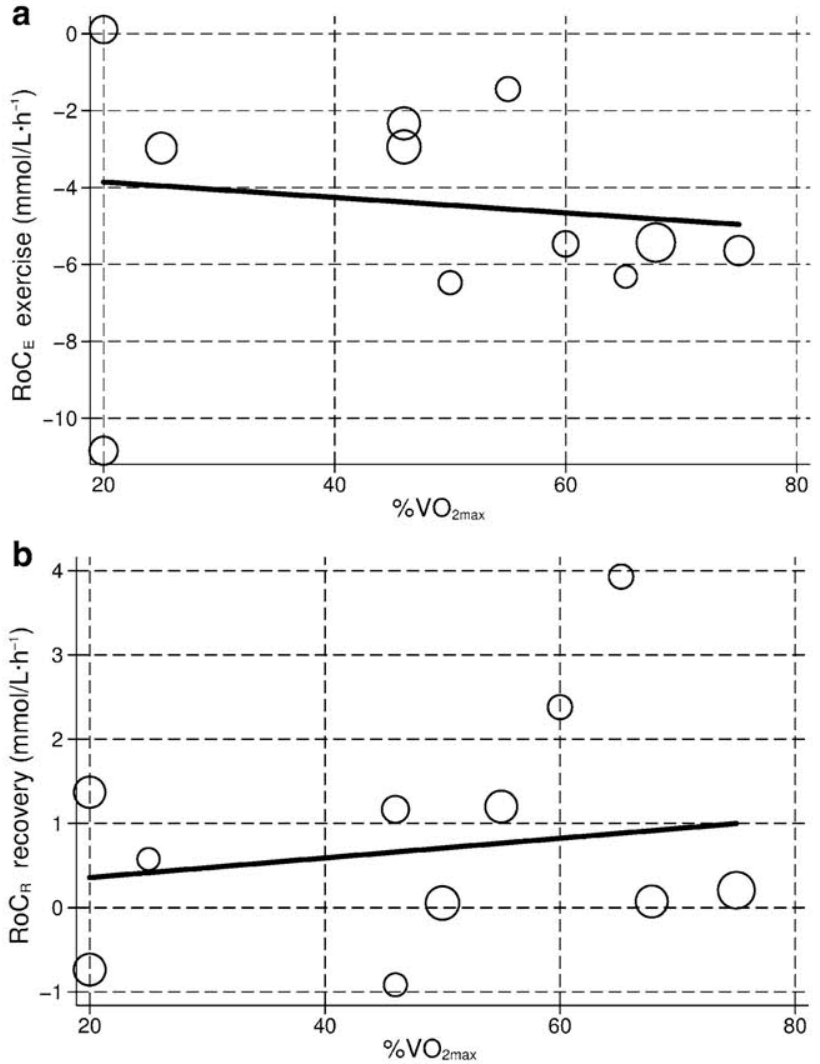

Fig. 8 Dose/response analysis for the influence of exercise intensity, as expressed by $\% V \mathrm{O}_{2 \max }$, on the rate-of-change magnitudes $R o C_{E}$ (a) and $R_{0} C_{R}$ (b) for continuous physical activity. CONT continuous physical activity, $R o C_{E}$ glycemia rate-of-change during exercise, $R o C_{R}$ glycemia rate-of-change at recovery, $V O_{2 \max }$ maximal oxygen uptake

during exercise and in the immediate recovery stage. Average RoC values during exercise and recovery phases, as well as their corresponding $95 \%$ CIs, were estimated by detrending within-study glycemic variations over time with respect to resting reference profiles. Sub-analyses between specific exercise categories were also conducted.

We found that, during CONT exercise at moderate intensities (range 20-75 \% VO 2 max ), glucose concentration declined at a rapid rate when compared with resting periods $\left(\mathrm{RoC}_{\mathrm{E}} \quad\{\mathrm{CONT}\right.$ vs. $\mathrm{REST}\}=-4.43 \mathrm{mmol} / \mathrm{L} \mathrm{h}^{-1}$ on average) and slowly reverted after the bout concluded (mean $\mathrm{RoC}_{\mathrm{R}}\{\mathrm{CONT}$ vs. REST $\}=+0.70 \mathrm{mmol} / \mathrm{L} \mathrm{h}^{-1}$ ). 
These results are in reasonable concordance with RoC during exercise as reported by Dubé et al. [20,21] before rescue dextrose was infused intravenously in their experiments. In particular, Dubé et al. [20] documented $\mathrm{RoC}_{\mathrm{E}}$ equaling $-4.8 \pm 1.2,-6.3 \pm 1.2$, and $-3.6 \pm 0.6 \mathrm{mmol} /$ $\mathrm{L} \mathrm{h}^{-1}$ (expressed as mean \pm standard error of the mean [SEM]) for trial arms with 0,15 , or $30 \mathrm{~g}$ of carbohydrate supplements pre-exercise, respectively; whereas Dubé et al. [21] reported $R_{0} C_{E}$ values of $-9.6 \pm 2.4$ and $-6.0 \pm 1.2 \mathrm{mmol} / \mathrm{L} \mathrm{h}^{-1}$ (mean $\pm \mathrm{SEM}$ ) for their early and late postprandial exercise arms.

Decreases in glycemia for RESIST physical activity were milder than in the case of CONT exercise, both with respect to REST reference $\left(\mathrm{RoC}_{\mathrm{E}}\right.$ \{RESIST vs. $\operatorname{REST}\}=-2.61 \mathrm{mmol} / \mathrm{L} \mathrm{h}^{-1}$ on average, against mean $\operatorname{RoC}_{\mathrm{E}}\{\mathrm{CONT}$ vs. REST $\}=-4.43 \mathrm{mmol} / \mathrm{L} \mathrm{h}^{-1}$ ); and in the direct comparison $\left(\operatorname{RoC}_{\mathrm{E}}\right.$ \{RESIST vs. CON$\left.\mathrm{T}\}=+2.86 \mathrm{mmol} / \mathrm{L} \mathrm{h}^{-1}\right)$. Likewise, recovery rates were slower for RESIST.

In the case of IHE exercise, discrepancies arose for the quantitative comparisons. $\mathrm{Ro}_{\mathrm{E}}$ values calculated with respect to REST reference, based on two studies, yielded very pronounced decays $\left(\operatorname{RoC}_{\mathrm{E}}\{\mathrm{IHE}\right.$ vs. REST $\}$ $=-5.25 \mathrm{mmol} / \mathrm{L} \mathrm{h}^{-1}$ on average) versus the comparatively lower absolute values for CONT (mean RoC $\mathrm{C}_{\mathrm{E}}$ $\{\mathrm{CONT}$ vs. $\mathrm{REST}\}=-4.43 \mathrm{mmol} / \mathrm{L} \mathrm{h}^{-1}$ ); whereas analyses of IHE directly versus CONT indicated slower glucose decreases for IHE $\left(\mathrm{RoC}_{\mathrm{E}} \quad\{\mathrm{IHE}\right.$ vs. CON$\mathrm{T}\}=+1.57 \mathrm{mmol} / \mathrm{L} \mathrm{h}^{-1}$ ), based on three studies.

\subsection{Strengths and Limitations}

We performed a comprehensive systematic literature review, identifying ten studies as relevant to our metaanalysis. Of note, we presented a novel methodology to evaluate quantitatively acute trends in glycemia by approximating variations in linear segments and by computing average RoC for exercise and recovery stages. By recording fluctuations in the reference profiles and subtracting them out at a study level prior to the pooling, we aimed to mitigate background trends due to factors other than exercise itself in each particular trial, and hence reduce bias. Three exercise types were included, namely, continuous physical activity at moderate intensity, IHE, and resistance exercise.

Limitations to this analysis need to be considered. First, our meta-regression to ascertain the dose/response relationship to varying exercise intensities in $\mathrm{RoC}_{\mathrm{E}}$ for CONT showed more pronounced decays for increasing load. This conclusion should nonetheless be restricted to the range of intensities under analysis here $\left(20-75 \% V_{2} \mathrm{O}_{2 \max }\right)$, i.e. moderate exertion. Very vigorous exercise $(>80 \%$ $\left.V \mathrm{O}_{2 \max }\right)$ has been reported to induce post-exercise hyperglycemia in type 1 diabetes due to catecholamine responses causing 7- to 8 -fold rises in glucose production that are not matched by glucose utilization, which increases 3- to 4-fold [47-49].

We encountered a quantitative discrepancy regarding the magnitude of exercise effects on $\mathrm{RoC}_{\mathrm{E}}$ for IHE as compared with CONT. In the analyses with REST as reference (see Fig. 4), the aggregate of two IHE studies yielded $\operatorname{RoC}_{\mathrm{E}} \quad\{\mathrm{IHE}$ vs. REST $\}=-5.25 \mathrm{mmol} / \mathrm{L} \mathrm{h}^{-1}$ $(95 \% \mathrm{CI}-7.02$ to -3.48$] \mathrm{mmol} / \mathrm{L} \mathrm{h}^{-1}, I^{2} 0 \%$ ) versus a comparatively more restricted decay for CONT: $\mathrm{RoC}_{\mathrm{E}}$ $\{\mathrm{CONT}$ vs. REST $\}=-4.43 \mathrm{mmol} / \mathrm{L} \mathrm{h}^{-1}(95 \% \mathrm{CI}-6.06$ to $-2.79 \mathrm{mmol} / \mathrm{L} \mathrm{h}^{-1}, I^{2} 41 \%$ ) calculated based on seven studies and 11 comparisons (see Fig. 3). Conversely, the direct confrontation (see Fig. 6) resulted in $\mathrm{RoC}_{\mathrm{E}}$ \{IHE vs. $\mathrm{CONT}\}=+2.86 \mathrm{mmol} / \mathrm{L} \mathrm{h}^{-1}(95 \% \mathrm{CI}-1.49$ to +7.20 ; $I^{2} 41 \%$ ), with three studies involved; hence pointing to a slower decline in glycemia for IHE than for CONT ( $p=0.15$, not significant). The scarcity of available studies involving IHE (four in total [29-31, 33], with Iscoe and Riddell [31] presenting REST, CONT, and IHE trial arms) may explain this shortcoming to some extent. We also encountered statistical heterogeneity $\left(I^{2} 53 \%\right.$ for $\mathrm{RoC}_{\mathrm{E}}$ $\{$ IHE vs. CONT $\}$ ) and a substantial methodological diversity among study protocols; in particular, in the definition of the IHE session. Guelfi et al. [29] utilized intermittent $4 \mathrm{~s}$ short bursts by maximal sprints every $2 \mathrm{~min}$, with subjects remaining seated without physical activity between sprints-i.e. passive recovery. In another study [30], the same investigators defined a different protocol, in which periods between maximal sprints corresponded to sustained physical activity at $40 \% V \mathrm{O}_{2 \max }$. Maran et al. [33] utilized submaximal sprints $\left(85 \% V^{2} \mathrm{O}_{2 \max }\right)$ with a duration of $5 \mathrm{~s}$ and repeated every $2 \mathrm{~min}$. In an even more diverse protocol, Iscoe and Riddell [31] compared CONT at sustained $55 \% V_{2 \max }$ versus IHE with sustained $50 \%$ $V \mathrm{O}_{2 \max }$ plus $15 \mathrm{~s}$ maximal sprints every $5 \mathrm{~min}$, aiming for identical mechanical work for both tasks. Regarding glucose variations, Guelfi et al. [30] documented a greater decline in absolute terms-i.e. not accounting for RoCsfor CONT $(-4.4 \pm 1.2 \mathrm{mmol} / \mathrm{L}$ in $45 \mathrm{~min}$, mean $\pm \mathrm{SD})$ versus IHE $(-2.9 \pm 0.8 \mathrm{mmol} / \mathrm{L})$, with statistical significance $(p=0.006)$; whereas Maran et al. [33] observed glycemia values that tended to be higher after IHE, but not significantly so. Conversely, Iscoe and Riddell [31] reported virtually identical glycemic profiles throughout the CONT and IHE bouts and in recovery until $2 \mathrm{~h} 15 \mathrm{~min}$ post-exercise, with noticeable differences in nocturnal levels: increased risk of nocturnal hypoglycemia $<4 \mathrm{mmol} /$ $\mathrm{L}$ in the CONT trial arm (two hypoglycemia events per night in REST, compared with five events in CONT and three in IHE). Interestingly, these findings manifestly contradict those by Maran et al. [33], who reported two 
nocturnal hypoglycemia events $<3.33 \mathrm{mmol} / \mathrm{L}$ in CONT, against seven events for IHE $(p<0.05)$. In summary, evidence appears to be conflicting in literature regarding IHE effects in type 1 diabetes; further research in this direction may be needed.

We found a number of studies that, had we incorporated them, may have increased the statistical power of our analyses. However, we decided not to do so due to a dissimilar approach in these articles [41-46], which focused on determining the effectiveness of auxiliary interventions to manage glucose excursions caused by exercise: modifications of insulin regimes to accommodate exercise (insulin analogs [41], pump cessation [42], bolus reductions [43], etc.) or food supplementations [44 46]. Given the aim of these experiments, the auxiliary intervention was either applied or not, but subjects exercised in both trial arms. Therefore, we could not have subtracted inherent within-study background spurious trends in glycemia to avoid introducing bias.

Several other aspects of potential relevance were not accounted for in our analyses. Method for glucose measurement is one of these variables. Blood sampling, used in seven of ten studies [28, 32-37], constitutes the most accurate and reliable technique. Capillary samples, which were obtained in two studies $[29,30]$, are more prone to error and delays than venous blood determinations. It should be noted that Guelfi et al. [29, 30] also collected venous blood samples, but these were used to measure free insulin, glucagon, growth hormones, etc., not to determine glycemia. The third alternative, continuous glucose monitoring (CGM), has, in principle, lower accuracy than venous or capillary measurements. Nonetheless, it was the technique of choice for Iscoe and Riddell [31]. Yardley et al. [28] used CGM in addition to blood samples, in order to assess the accuracy achieved by CGM sensors under exercise circumstances. However, the data from Yardley et al. [28] included in our meta-analysis were obtained from blood measurements only. According to Yardley et al. [28], CGM underestimated plasma glucose considerably at $\operatorname{REST}(-1.29 \pm 1.39 \mathrm{mmol} / \mathrm{L}$, mean $\pm \mathrm{SD}, p<0.001)$, to a lower extent during RESIST $(-0.71 \pm 1.35 \mathrm{mmol} / \mathrm{L}$, $p<0.001$ ) and with non-significant errors during CONT exercise $(-0.11 \pm 1.71 \mathrm{mmol} / \mathrm{L}, p=0.416)$. On the contrary, CGM was associated with substantial errors during exercise for pregnant women with type 1 diabetes [50] (18.4\% error with respect to plasma glucose during exercise-brisk walking-vs. $11.8 \%$ at rest, $p<0.001$ ). Of note, the study by Kumareswaran et al. [50] reported results consistent with our meta-analysis: a decay of $24.6 \%$ in terms of relative $\mathrm{RoC}$ for exercise vs. $12.3 \%$ in sedentary situations $(p<0.001)$.

Glycemia level at the onset of the physical activity session may have also acted as a confounder. Jenni et al.
[51] conducted a glucose clamp experiment and showed that the rate of carbohydrate oxidation was higher in exercise performed under hyperglycemia conditions, whereas lipid oxidation was higher in the euglycemia clamp. Consequently, more pronounced falls could be expected if physical activity was commenced with high glucose values. This was the case for the majority of the included studies, for which exercise took place with concentrations around $10 \mathrm{mmol} / \mathrm{L}$ or above: Soo et al. [36] (approximate range $12-13 \mathrm{mmol} / \mathrm{L}$ ), Rabasa-Lhoret et al. [35] $\left(50 \% \quad V \mathrm{O}_{2 \max }\right.$ trial arm $[10.7 \pm 0.7 \mathrm{mmol} / \mathrm{L}$, mean $\pm \mathrm{SEM}])$, Yamanouchi et al. [37] $(\sim 10 \mathrm{mmol} / \mathrm{L}$ pre-prandial and $\sim 15 \mathrm{mmol} / \mathrm{L}$ post-prandial), Peter et al. [34] (approximate range 11-12 $\mathrm{mmol} / \mathrm{L}$ ), Guelfi et al. [29] $(10.9 \pm 1.9 \mathrm{mmol} / \mathrm{L}$ for REST and $11.0 \pm 1.8 \mathrm{mmol} / \mathrm{L}$ for IHE, mean \pm SD), Guelfi et al. [30] $(11.0 \pm 2.3 \mathrm{mmol} / \mathrm{L}$ for CONT and $11.5 \pm 3.9 \mathrm{mmol} / \mathrm{L}$ for IHE, mean $\pm \mathrm{SD}$ ), and Yardley et al. [28] (CONT trial arm [ $\sim 10 \mathrm{mmol} / \mathrm{L}])$. Other studies commenced at more restrained glycemia levels: Jankovec et al. [32] (approximate range 7-8 mmol/ L), Rabasa-Lhoret et al. [35] $(8.8 \pm 0.55 \mathrm{mmol} / \mathrm{L}$ for their $25 \% \quad V \mathrm{O}_{2 \max }$ trial and $8.5 \pm 1.3 \mathrm{mmol} / \mathrm{L}$ for $75 \%$ $V \mathrm{O}_{2 \max }$, mean $\pm \mathrm{SEM}$ ), and Yardley et al. [28] (RESIST trial $\mathrm{arm} \sim 8.5 \mathrm{mmol} / \mathrm{L}$ ). Information in this regard was not provided in Maran et al. [33], whereas Iscoe and Riddell [31] mentioned an absolute fall of approximately $-5 \mathrm{mmol} / \mathrm{L}$ and $\sim 50 \%$ relative decay, although data were not reported explicitly in either text or graphs. The most marked decay rates among the included publications were reported, in this order, by Yamanouchi et al. [37] (postbreakfast exercise trial arm), Rabasa-Lhoret et al. [35] (50\% VO ${ }_{2 \max }$ trial), Peter et al. [34], Rabasa-Lhoret et al. [35] $\left(75 \% V_{\mathrm{O}_{2 \max }}\right.$ trial), and Yardley et al. [28] (CONT). In view of these data, there does not appear to be an evident direct relationship between the highest blood glucose concentrations at exercise onset and the most substantial RoC $_{\mathrm{E}}$ values.

Plasma insulin concentrations during exercise may have also had an important role in the glucoregulatory response and have impacted our analysis as a confounder. In a euglycemia clamp experiment (glucose fixed at approximately $8 \mathrm{mmol} / \mathrm{L}$ ) under two hyperinsulinemic regimens at different levels (plasma insulin at $\sim 150$ or $\sim 540 \mathrm{pmol} / \mathrm{L}$, corresponding to typical pre- and postprandial concentrations in patients with type 1 diabetes), Chokkalingam et al. [52] studied whole-body and muscle metabolism in exercise. Markedly higher exogenous glucose utilization was observed in the trial arm at $540 \mathrm{pmol} / \mathrm{L}$. However, the amount of muscle glycogen utilized in both situations was similar, and carbohydrate oxidation rates were only around $15 \%$ more in the trial arm with the highest insulinemia. Consequently, the influence of distinct plasma insulin levels in otherwise equivalent exercise conditions remains 
unclear, as outlined by Chokkalingam et al. [52]. Regarding the studies included here, three articles $[28,31,33]$ did not provide experimental data on insulin concentration. In the following three cases, patients exercised at levels lower than both conditions in Chokkalingam et al. [52]: Jankovec et al. [32] (average insulinemia $\sim 80 \mathrm{pmol} / \mathrm{L}$ and great inter-subject variability, without statistical differences versus REST); Soo et al. [36] (basal $84 \pm 18 \mathrm{pmol} / \mathrm{L}$, mean \pm SEM; authors reported no significant correlation between basal free insulin and glycemic response); and Yamanouchi et al. [37] (pre-prandial trial arm $[55.3 \pm 21.5 \mathrm{pmol} / \mathrm{L}$, mean $\pm \mathrm{SD}])$. Peter et al. [34] documented an average plasma insulin of $\sim 300 \mathrm{pmol} / \mathrm{L}$ during both REST and CONT, without statistical differences between trials in terms of area under the curve for insulinemia $(p=0.116)$. Physical activity bouts in the remaining studies took place with values comparable to the $150 \mathrm{pmol} / \mathrm{L}$ selected in Chokkalingam et al. [52]: Rabasa-Lhoret et al. [35] (insulin bolus $90 \mathrm{~min}$ prior to exercise onset, peak insulinemia at $188.5 \pm 28.0 \mathrm{pmol} / \mathrm{L}$, mean $\pm \mathrm{SD}$; peak occurring $30 \mathrm{~min}$ pre-exercise), Yamanouchi et al. [37] (postprandial trial [insulin bolus $90 \mathrm{~min}$ prior to exercise onset, peak at $231.9 \pm$ $162.3 \mathrm{pmol} / \mathrm{L}$, mean $\pm \mathrm{SD}$ ), Guelfi et al. [29] (IHE exercise commenced at $198.1 \pm 148.0 \mathrm{pmol} / \mathrm{L}$, mean \pm SD; no statistical difference with respect to REST), and Guelfi et al. [30] (IHE and CONT exercise commenced, respectively, at approximately 160 and $140 \mathrm{pmol} / \mathrm{L}$; no statistical differences in insulinemia profiles at any point of exercise or recovery).

We did not consider time of the day at which exercise was performed, although it may have also influenced outcomes. In a euglycemic clamp in which exercise was performed in the afternoon (4 p.m.), MacMahon et al. [53] showed that glucose infusion rates necessary to maintain stable glycemia peaked in a biphasic manner: during exercise and early recovery, plus in the night afterwards (midnight to 4 a.m.). Conversely, in an otherwise equivalent design with exercise performed at midday, Davey et al. [54] did not observe the same biphasic behavior in the glucose infusion rates, which were elevated for $11 \mathrm{~h}$ postexercise. It is difficult to draw solid conclusions in this regard from the included studies: all but three experiments were carried out in the morning; with the exceptions of Maran et al. [33] (exercise at approximately 2 p.m.), Iscoe and Riddell [31], and Yardley et al. [28] (both at 5 p.m).

Only five of the included studies provided explicit information on participants' degree of fitness or prior physical training status (see Table 1). This aspect may have had an effect on the glucoregulatory response, including glucose uptake into skeletal muscle, even at a fixed relative intensity-i.e. the same $\% V \mathrm{O}_{2 \max }$ [55]. Moreover, the population studied by Guelfi et al. [29] consisted of adolescents, whose hormonal response to physical activity may differ from that of adults [56].

\subsection{Implications for Practice and Research}

Better understanding of the acute glycemic effects of physical activity is of considerable importance to clinicians and patients with type 1 diabetes aiming for a tighter management of acute, exercise-related glucose excursions. Currently, guidelines for exercising with type 1 diabetes are based on small studies or observational evidence.

Our systematic review confirmed the known glucoselowering effects of moderate physical activity under various circumstances. Uniquely, we quantified trends in blood glucose by means of two RoC magnitudes: RoC $\mathrm{C}_{\mathrm{E}}$, $\mathrm{RoC}_{\mathrm{R}}$ during and after exercise. Quantitative information presented here (mean RoCs and $95 \%$ CIs) may be useful when advising patients on strategies to maintain optimal glucose control and avoid post-exercise hyperand especially hypoglycemia, improving safety and quality of life for physically active people with type 1 diabetes.

Our review also identified the lack of parallel controlled studies comparing physiological responses to different exercise categories. In addition, we encountered conflicting evidence regarding effects of IHE physical activity in subjects with type 1 diabetes. More homogeneous IHE exercise protocols (particularly in terms of sprint duration, frequency of repetition, and intensity) and further research may be needed.

\subsection{Comparison with Previous Reviews}

Tonoli et al. [57] recently analysed the overall effect on glycemic control of a single bout of physical activity, based on the pooling of 15 acute exercise studies: nine aerobic and six IHE. Authors also surveyed the impact on glycosylated hemoglobin $\left(\mathrm{HbA}_{1 \mathrm{c}}\right)$ of regular/chronic training for up to several months. In this study [57], Cohen's $d$ statistic was used as the main outcome to characterize the glucoregulatory impact of physical activity. Overall, Tonoli et al. [57] reported substantial decreases in venous glucose levels due to acute aerobic exercise in adults $(-6.0$ mean Cohen's $d$ value; $95 \% \mathrm{CI}-6.87$ to -5.14 ), these reductions being considerably larger than those for acute IHE activity $(-4.35 ; 95 \% \mathrm{CI}-6.41$ to -2.65 for Cohen's d). While these results from this study [57] are in qualitative agreement with our findings, quantitative comparison with our work is not feasible because Cohen's $d$ is a dimensionless measure that reflects the average difference in a relative manner, i.e. normalized by the SD in each study [58]. In contrast, and as a major novel contribution of this review, we addressed glucose variations in absolute 
terms via the $\mathrm{RoC}_{\mathrm{E}}, \mathrm{RoC}_{\mathrm{R}}$ RoC. We considered these RoC magnitudes (expressed in tangible units: $\mathrm{mmol} / \mathrm{L} \mathrm{h}^{-1}$ ) to be a more accessible, straightforward estimation of exercise-related glucose dynamics, and hence more easily translated into clinical practice and patients' self-management. In addition, we extended the analysis by Tonoli et al. [57] of glucose dynamics by incorporating variations during the early recovery stage.

Tonoli et al. [57] agree with our discussion regarding the limitations of available literature, pointed out the difficulty in pooling studies due to marked discrepancies in terms of exercise protocols, and advocate for more standardization and broader samples of subjects.

\section{Conclusions}

In this review we conducted a quantitative analysis of the acute impact of physical activity on the glucoregulatory system in type 1 diabetes, by means of novel RoC magnitudes to characterize numerically how glycemia varies during exercise and immediately afterwards (early recovery). We found that, for CONT at moderate intensities, glycemia declined rapidly at an average rate of $\mathrm{RoC}_{\mathrm{E}}$ $\{$ CONT vs. REST $\}=-4.43 \mathrm{mmol} / \mathrm{L} \mathrm{h}^{-1}$ and mildly recovered at $\operatorname{RoC}_{\mathrm{R}}\{\mathrm{CONT}$ vs. REST $\}=+0.70 \mathrm{mmol} /$ $\mathrm{L} \mathrm{h}^{-1}$. RESIST showed more constrained average decays and recoveries than CONT, $\mathrm{RoC}_{\mathrm{E}}\{$ RESIST vs. CONT $\}=$ $+2.86 \mathrm{mmol} / \mathrm{L} \mathrm{h}^{-1}$ and $\operatorname{RoC}_{\mathrm{R}}$ \{RESIST vs. CONT $\}=$ $-2.40 \mathrm{mmol} / \mathrm{L} \mathrm{h}^{-1}$; whereas discrepancies were encountered regarding the magnitude of IHE decreases in glycemia with respect to CONT, either directly compared or via the REST reference.

Acknowledgments The authors would like to thank the reviewers for their comments, which led to a substantial improvement of our work. In particular, their identification of the study by Yardley et al. [28] is of special note. This work was partly supported by a doctoral research Grant from the Technical University of Madrid, by a fellowship from the Spanish Ministry of Education, and by the 'APRIORI' Project (FIS PS09/01318) from the Spanish Ministry of Science and Innovation. Funders were not involved in the research or its publication. The authors declare that there is no conflict of interest that could be perceived as prejudicing the impartiality of the review reported.

\section{References}

1. American Diabetes Association. Physical activity/exercise and diabetes. Diabetes Care. 2004;27(S1):S58-62.

2. Nagi D, editor. Exercise and sport in diabetes, 2nd ed. UK: Wiley; 2006.

3. Ertl AC, Davis SN. Evidence for a vicious cycle of exercise and hypoglycemia in type 1 diabetes mellitus. Diabetes Metab Res Rev. 2004;20(2):124-30.
4. Cryer PE. Exercise-related hypoglycemia-associated autonomic failure in diabetes. Diabetes. 2009;58(9):1951-2.

5. Riddell MC, Burr J. Evidence-based risk assessment and recommendations for physical activity clearance: diabetes mellitus and related comorbidities. Appl Physiol Nutr Metab. 2011;36(S1): 154-89.

6. Sonnenberg GE, Kemmer FW, Berger M. Exercise in type 1 (insulin-dependent) diabetic patients treated with continuous subcutaneous insulin infusion-prevention of exercise induced hypoglycaemia. Diabetologia. 1990;33(11):696-703.

7. Marliss EB, Vranic M. Intense exercise has unique effects on both insulin release and its roles in glucoregulation: implications for diabetes. Diabetes. 2002;51(S):S271-S283.

8. Chu L, Hamilton J, Riddell MC. Clinical management of the physically active patient with type I diabetes. Phys Sports Med. 2011;39(2):64-77.

9. Riddell MC, Perkins B. Type 1 diabetes and vigorous exercise: applications of exercise physiology to patient management. Can J Diabetes. 2006;30(1):63-71.

10. Lumb AN, Gallen IW. Diabetes management for intense exercise. Curr Opin Endocrinol Diabetes Obes. 2009;16(2):150-5.

11. Guelfi KJ, Jones TW, Fournier PA. New insights into managing the risk of hypoglycaemia associated with intermittent highintensity exercise in individuals with type 1 diabetes mellitusimplications for existing guidelines. Sports Med. 2007;37(11): 937-46.

12. Jiménez $C$, Santiago $M$, Sitler $M$, et al. Insulin-sensitivity response to a single bout of resistive exercise in type 1 diabetes mellitus. J Sport Rehabil. 2009;18(4):564-71.

13. Harmer AR, Chisholm DJ, McKenna MJ, et al. High-intensity training improves plasma glucose and acid-base regulation during intermittent maximal exercise in type 1 diabetes. Diabetes Care. 2007;30(5): 1269-71.

14. Braken RM, West DJ, Stephens JW, et al. Impact of pre-exercise rapid-acting insulin reductions on ketogenesis following running in type 1 diabetes. Diabet Med. 2011;28(2):218-22.

15. Kilbride L, Charlton J, Aitken G, et al. Managing blood glucose during and after exercise in type 1 diabetes: reproducibility of glucose response and a trial of a structured algorithm adjusting insulin and carbohydrate intake. J Clin Nurs. 2011;20(23-24):3423-9.

16. Moher D, Liberati A, Tetzlaff J, et al. Preferred reporting items for systematic reviews and meta-analyses: the PRISMA statement. PLoS Med 2009;6(7):e1000097. doi:10.1371/journal.pmed. 1000097

17. Higgins JPT, Green S, editors. Cochrane handbook for systematic reviews of interventions version 5.1.0 [updated March 2011]. The Cochrane Collaboration, 2011. Available from http://www. cochrane-handbook.org.

18. Coletti MH, Bleich HL. Medical subject headings used to search the biomedical literature. J Am Med Inform Assoc. 2001;8(4): 317-23.

19. Review Manager (RevMan) [Computer program]. Version 5.2. Copenhagen: The Nordic Cochrane Centre, The Cochrane Collaboration; 2012.

20. Dubé MC, Weisnagel SJ, Prud'homme D, et al. Exercise and newer insulins: how much glucose supplement to avoid hypoglycemia? Med Sci Sports Exerc. 2005;37(8):1276-82.

21. Dubé MC, Weisnagel SJ, Prud'homme D, et al. Is early and late post-meal exercise so different in type 1 diabetic lispro users? Diabetes Res Clin Pract. 2006;72(2):128-34.

22. Dubé MC, Lavoie C, Weisnagel SJ. Glucose or intermittent highintensity exercise in glargine/glulisine users with T1DM. Med Sci Sports Exerc. 2013;45(1):3-7.

23. Yardley JE, Kenny GP, Perkins BA, et al. Effects of performing resistance exercise before versus after aerobic exercise on glycemia in type 1 diabetes. Diabetes Care. 2012;35(4):669-75. 
24. Yardley JE, Kenny GP, Perkins BA, et al. Resistance versus aerobic exercise: acute effects on glycemia in type 1 diabetes. Diabetes Care. 2013;36(3):537-42.

25. Bussau VA, Ferreira LD, Jones TW, et al. The 10-s maximal sprint: a novel approach to counter an exercise-mediated fall in glycemia in individuals with type 1 diabetes. Diabetes Care. 2006;29(3):601-6.

26. Bussau VA, Ferreira LD, Jones TW, et al. A 10-s sprint performed prior to moderate-intensity exercise prevents early postexercise fall in glycaemia in individuals with type 1 diabetes. Diabetologia. 2007;50(9):1815-8.

27. Tsalikian E, Mauras N, Beck RW, et al. Impact of exercise on overnight glycemic control in children with type 1 diabetes mellitus. J Pediatr. 2005;147(4):528-34.

28. Yardley JE, Sigal RJ, Kenny GP, et al. Point accuracy of interstitial continuous glucose monitoring during exercise in type 1 diabetes. Diabetes Technol Ther. 2013;15(1):46-9.

29. Guelfi KJ, Jones TW, Fournier PA. Intermittent high-intensity exercise does not increase the risk of early postexercise hypoglycemia in individuals with type 1 diabetes. Diabetes Care. 2005;28(2):416-8.

30. Guelfi KJ, Jones TW, Fournier PA. The decline in blood glucose levels is less with intermittent high-intensity compared with moderate exercise in individuals with type 1 diabetes. Diabetes Care. 2005;28(6): 1289-94.

31. Iscoe KE, Riddell MC. Continuous moderate-intensity exercise with or without intermittent high-intensity work: effects on acute and late glycaemia in athletes with type1 diabetes mellitus. Diabet Med. 2011;28(7):824-32.

32. Jankovec Z, Krcma M, Gruberova J, et al. Influence of physical activity on metabolic state within a 3-h interruption of continuous subcutaneous insulin infusion in patients with type 1 diabetes. Diabetes Technol Ther. 2011;13(12):1234-9.

33. Maran A, Pavan P, Bonsembiante B, et al. Continuous glucose monitoring reveals delayed nocturnal hypoglycemia afterintermittent high-intensity exercise in nontrained patients with type 1 diabetes. Diabetes Technol Ther. 2010;12(10):763-8.

34. Peter R, Luzio SD, Dunseath G, et al. Effects of exercise on the absorption of insulin glargine in patients with type 1 diabetes. Diabetes Care. 2005;28(3):560-5.

35. Rabasa-Lhoret R, Bourque $\mathbf{J}$, Ducros $\mathbf{F}$, et al. Guidelines for premeal insulin dose reduction for postprandial exercise of different intensities and durations in type 1 diabetic subjects treated intensively with a basal-bolus insulin regimen (ultralente-lispro). Diabetes Care. 2001;24(4):625-30.

36. Soo K, Furler SM, Samaras K, et al. Glycemic responses to exercise in IDDM after simple and complex carbohydrate supplementation. Diabetes Care. 1996;19(6):575-9.

37. Yamanouchi $\mathrm{K}$, Abe R, Takeda A, et al. The effect of walking before and after breakfast on blood glucose levels in patients with type 1 diabetes treated with intensive insulin therapy. Diabetes Res Clin Pract. 2002;58(1):11-8.

38. da Cunha FA, Farinatti PTV, Midgley AW. Methodological and practical application issues in exercise prescription using the heart rate reserve and oxygen uptake reserve methods. J Sci Med Sport. 2011;14(1):46-57.

39. Gaskill SE, Bouchard C, Rankinen T, et al. \%Heart rate reserve is better related to $\% V \mathrm{O}_{2 \max }$ than to $\% V \mathrm{O}_{2 \text { reserve: }}$ the HERITAGE family study. Med Sci Sports Exerc. 2004;36(5):S3.

40. Rotstein A, Meckel Y. Estimation of $\% V \mathrm{O}_{2}$ reserve from heart rate during arm exercise and running. Eur J Appl Physiol. 2000;83(6):545-50.

41. Arutchelvam V, Heise T, Dellweg $S$, et al. Plasma glucose and hypoglycaemia following exercise in people with type 1 diabetes: a comparison of three basal insulins. Diabet Med. 2009;26(10): 1027-32.

42. Tsalikian E, Fox L, Janz KF, et al. Prevention of hypoglycemia during exercise in children with type 1 diabetes by suspending basal insulin. Diabetes Care. 2006;29(10):2200-4.

43. West DJ, Morton RD, Bain SC, et al. Blood glucose responses to reductions in pre-exercise rapid-acting insulin for $24 \mathrm{~h}$ after running in individuals with type 1 diabetes. J Sports Sci. 2010;28(7):781-8.

44. Dubé MC, Lavoie C, Galibois I, et al. Nutritional strategies to prevent hypoglycemia at exercise in diabetic adolescents. Med Sci Sports Exerc. 2012;44(8):1427-32.

45. Perrone C, Laitano O, Meyer F. Effect of carbohydrate ingestion on the glycemic response of type 1 diabetic adolescents during exercise. Diabetes Care. 2005;28(10):2537-8.

46. West DJ, Stephens JW, Bain SC, et al. A combined insulin reduction and carbohydrate feeding strategy $30 \mathrm{~min}$ before running best preserves blood glucose concentration after exercise through improved fuel oxidation in type 1 diabetes mellitus. J Sports Sci. 2011;29(3):279-89.

47. Marliss EB, Vranic M. Intense exercise has unique effects on both insulin release and its roles on glucoregulation-implications for diabetes. Diabetes. 2002;51(Suppl. 1):S271-83.

48. Sigal RJ, Fisher SJ, Manzon A, et al. Glucoregulation during and after intense exercise: effects on $\alpha$-adrenergic blockade. Metabolism. 2000;49(3):386-94.

49. Sigal RJ, Fisher SJ, Halter JB, et al. Glucoregulation during and after intense exercise: effects on $\beta$-adrenergic blockade in subjects with type 1 diabetes mellitus. J Clin Endocrinol Metab. 1999;84:3961-71.

50. Kumareswaran K, Elleri D, Allen JM, et al. Accuracy of continuous glucose monitoring during exercise in type 1 diabetes pregnancy. Diabetes Technol Ther. 2013;15(3):223-9.

51. Jenni S, Oetliker C, Allemann S, et al. Fuel metabolism during exercise in euglycaemia and hyperglycaemia in patients with type 1 diabetes mellitus - a prospective single-blinded randomised crossover trial. Diabetologia. 2008;51:1457-65.

52. Chokkalingam K, Tsintzas K, Norton L, et al. Exercise under hyperinsulinaemic conditions increases whole-body glucose disposal without affecting muscle glycogen utilisation in type 1 diabetes. Diabetologia. 2007;50:414-21.

53. MacMahon SK, Ferreira LD, Ratnam N, et al. Glucose requirements to maintain euglycemia after moderate-intensity afternoon exercise in adolescents with type 1 diabetes are increased in a biphasic manner. J Clin Endocrinol Metab. 2007;92(3):963-8.

54. Davey RJ, Howe W, Paramalingam N, et al. The effect of midday moderate-intensity exercise on postexercise hypoglycemia risk in individuals with type 1 diabetes. J Clin Endocrinol Metab. 2013;98(7):2908-14.

55. Fujimoto T, Kemppainen J, Kalliokoski KK, et al. Skeletal muscle glucose uptake response to exercise in trained and untrained men. Med Sci Sports Exerc. 2003;35(5):777-83.

56. Riddell MC. The endocrine response and substrate utilization during exercise in children and adolescents. J Appl Physiol. 2008;105(2):725-33.

57. Tonoli C, Heyman E, Roelands B, et al. Effects of different types of acute and chronic (training) exercise on glycaemic control in type 1 diabetes mellitus: a meta-analysis. Sports Med. 2012; 42(12): 1059-80.

58. Cohen J. Statistical power analysis for the behavioral sciences, 2nd ed. USA: Lawrence Erlbaum Assoc. Inc.; 1988. 